\title{
INVESTIGACIONES
}

\section{Rasgos y tendencias de la Didáctica con TIC: retos a partir de la nueva ecología del aprendizaje}

\author{
Traits and trends of Teaching with ICT: \\ challenges from the new ecology of learning
}

\author{
Isabel Jiménez Becerra ${ }^{a}$ \\ ${ }^{a}$ Centro de Tecnologías para la Académica, Universidad de La Sabana, \\ Proyecto de Investigación Didácticas Innovadoras mediadas por TIC. Chía, Colombia. \\ isabel.jimenez@unisabana.edu.co
}

\begin{abstract}
RESUMEN
El artículo presenta los rasgos, tendencias y retos a los que se está enfrentado el profesorado en sus prácticas de enseñanza del siglo XXI a partir de los nuevos nichos de aprendizaje existentes. Esto ha llevado a plantear didácticas emergentes que se robustecen gracias al uso de las Tecnologías de la Información y la Comunicación (TIC), siendo su papel un andamiaje posible. Los retos planteados en el proyecto de investigación Didácticas Innovadoras mediadas por TIC han sido implementados en cincuenta y seis instituciones educativas colombianas donde se buscó identificar las formas de enseñanza existentes, así como redimensionar el papel de las didácticas (Jiménez-Becerra, I., 2018a) a partir de diversas investigaciones implementados por más de setenta profesores. Los diseños de investigación implementados en estos proyectos se clasifican en aquellos de corte básico (Investigación Acción y Estudio de Caso) así como aplicada (modelos de evaluación como CIPP y Guskey). Se presenta los principales resultados compilados a partir de los tres retos que caracterizan el proyecto: la redimensión de las estrategias de enseñanza, las concepciones del currículo y el pensamiento del profesorado sobre el uso de las TIC.

Palabras claves: didácticas, innovación, currículo, estrategias, aprendizaje, TIC.
\end{abstract}

\begin{abstract}
The article presents the features, trends and challenges that teachers are facing in their teaching practices of the 21 st century from the new existing learning niches. This has led to raise emerging didactics that are strengthened thanks to the use of Information and Communication Technologies (ICT), their role being a possible scaffolding. The challenges posed in the project of research Innovative Didactics mediated by ICT have been implemented in fifty-six Colombian educational institutions where it was sought to identify existing forms of teaching, as well as resize the role of didactics from various research implemented by more than seventy teachers. The research designs implemented in these projects are classified into those of basic cut (Action Research and Case Study) as well as applied (evaluation models such as CIPP and Guskey). The main results compiled from the three challenges that characterize the project are presented: the redimensión of the teaching strategies, the conceptions of the curriculum and the teachers' thinking on the use of ICT.
\end{abstract}

Keywords: didactics, innovation, curriculum, strategies, learning, ICT. 


\section{INTRODUCCIÓN}

Uno de los grandes retos que tiene el profesorado para afrontar con eficacia las nuevas circunstancias educativas, centradas en el desarrollo acelerado de la ciencia y el desarrollo de las Tecnologías de la Información y la Comunicación (en adelante TIC), está en reformular su saber didáctico.

Uno de los Caminos posibles para lograr dicha reformulación son las propias TIC. No obstante, lo que ha demostrado las diversas investigaciones mapeadas dentro de este proyecto de investigación ${ }^{1}$ es que las TIC, tal como lo plantea Ferran Ruíz Tarrogó en su artículo Escenarios de futuro y transformación del sistema educativo: "escaso impacto en la renovación de la organización y en las competencias y la evaluación del alumnado. Las TIC se conciben ante todo como instrumentos del profesorado, para reforzar la didáctica, y como herramientas de los alumnos (hacer trabajos, buscar información), pero el examen es tradicional o en todo caso las TIC se emplean para aplicar pruebas. Los profesores tienden a plantear el uso de las TIC como un asunto de opción y preferencia personal, y el énfasis se pone en la formación del profesorado" (2016, p. 103). En este contexto, lo que si se ha logrado identificar es el uso de las TIC como una herramienta más de trabajo que, en muchos de los casos, simplemente sustituye el tablero. Esto ha generado una regresión respecto a su función transformadora en la escuela, omitiendo los aspectos fundamentales que permiten responder a los nuevos nichos de aprendizaje en el marco de las necesidades de quien aprende. Autores como Guerrero (2014) plantea que el profesorado pierde de vista las condiciones que favorecen su uso en la enseñanza tales como la flexibilidad, la versatilidad, la interactividad y la conectividad, siendo el planteamiento de las estrategias didácticas el reto que debe enfrentar el profesorado para operatividad a dichas condiciones por medio de ambientes o materiales de aprendizaje mediados por TIC creados para tal alcance. Esto implica centrarse en plantear nuevos caminos que permitan reformular las prácticas de enseñanza usando las TIC. A partir de lo anterior, se hace necesario analizar cómo los profesores introducen las TIC en las aulas. Dentro de los rastreos generados en esta investigación se identificó un estudio llevado a cabo por Apple Classrooms of Tomorrow (1985) mencionado por Belloch (2012), en el cual se explica cómo la reformulación de las prácticas de enseñanza genera procesos de innovación por medio de investigaciones en el marco de los retos que asume la enseñanza con el uso de las TIC. Basado en estos resultados se han clasificado cinco tipos de necesidades en las instituciones que se han convertido en las fases de la innovación: acceso (gestión para instalar de las TIC en las instituciones), adopción (apoyo de las TIC para transformar los estilos de enseñanza), adaptación (integración de las TIC a las prácticas de enseñanza para enfrentar las nuevas necesidades de aprendizaje), apropiación (uso de las TIC para fortaleces las experiencias de aprendizaje a partir del trabajo colaborativo, creativo e interdisciplinario) e invención (se descubren nuevos usos de la tecnología y se combinan con otros usos de forma creativa). Tal como lo plantea Ruíz (2016), uno de los orígenes por los cuales la reformulación de las didácticas usando TIC puede estar en la limitación de la financiación, con lo que esta opción podría ser presentada ventajosamente a la opinión pública en nombre del realismo, del

Los trabajos que serán referenciados en este artículo y que pertenecen a este macroproyecto de investigación pueden ser consultados de manera particular en la Base de Datos para Trabajos de Grado de la Universidad de la Sabana, conocida como Intellectum. Se puede acceder a este espacio a través del siguiente link: https://intellectum.unisabana.edu.co/ handle/10818/9 
back to basics y del rendimiento escolar concebido en términos tradicionales. El presente artículo plantea los caminos posibles que se han abordado en el marco de investigación para lograr la reformulación de las prácticas de enseñanza y plantear así algunos caminos para alcanzar la innovación. Sus resultados son una postura emergente de la reformulación de la didáctica con uso de TIC, así como rutas posibles para implementar prácticas de enseñanza a partir de factores de transformación concebidos, en este proyecto, como "retos".

\section{RASGOS Y TENDENCIAS DE LA DIDÁCTICA CON TIC: RETOS PARA SU REFORMULACIÓN EN EL MARCO DE LA ECOLOGÍA DEL APRENDIZAJE}

En los últimos tiempos la escuela se enfrenta a reconfigurar el uso de las TIC cambiando el paradigma de su uso a partir de sus experiencias con la enseñanza y el aprendizaje. Este paradigma es clasificado por Kennedy (2008) quien lo establece a partir de diversas distinciones como: las tecnologías para "vivir" y las tecnologías para "aprender". Los adolescentes y jóvenes utilizan las primeras, pero tienen muy poca experiencia en el uso de la tecnología para el aprendizaje debido a que el profesorado no las implementa de manera regular para tal fin. Esto ha generado que la anhelada reformulación de las prácticas de enseñanza se ha quedado en promesas, pues lo resultados encontrados evidencian que la incorporación de las TIC como estrategia didáctica, mediación o andamiaje respecto a los procesos de enseñanza y aprendizaje se ha quedado en el campo instrumental alejando su implementación a una relación directa con la innovación. El uso de las tecnologías ha ido adquiriendo una dimensión más relacionada con el ocio y la comunicación que con el aprendizaje.

Un elemento clave que ha permitido plantear esta afirmación es cómo la incorporación de las TIC no ha sido las más adecuada, esto basado en que los resultados de las investigaciones no dan cuenta clara sobre el efecto e impacto del uso de las TIC en aspecto como el rendimiento académico o la cualificación en el aprendizaje basado en conocer qué se aprende y comprender el cómo se aprende mejor usando las TIC (Jiménez-Becerra, I., 2018b). En otras palabras, necesitamos entender cómo diseñar entornos (Jiménez-Becerra, I., Salamanca, L. \& López, L., 2018) y situaciones educativas que puedan mejorar el aprendizaje en el marco de los nichos de aprendizaje. Es así como aparece la necesidad de reformular del uso de las TIC en el marco de la didáctica, llamada a reformularse a partir de las posturas que plantean algunos de sus detractores quienes conciben su uso en la enseñanza como un recurso ${ }^{2}$ didáctico. Esta tendencia platea que su aporte en el aprendizaje es mínimo, pues está reducido a lo instrumental, al utilizar los computadores como maquinas que sustituyen la pizarra. En consecuencia, las TIC no han sido concebidas como la articulación de una estrategia donde los estudiantes se conviertan en verdaderos investigadores, dado que las herramientas tecnológicas potencializan el universo teórico, simbólico y conceptual de los mismos: nichos de aprendizaje.

Por su parte, se encuentran aquellos que reconocen el uso de las TIC como una necesidad y un desafío para la creatividad de la enseñanza. Es decir, como parte vital de

El término recurso o material, según San Martín (1991), se puede entender como aquellos artefactos que, en unos casos utilizando las diferentes formas de representación simbólica y en otros como referentes directos (objeto), incorporados en estrategias de enseñanza, contribuyen a la construcción del conocimiento, aportando significaciones parciales de los conceptos curriculares. 
la experiencia de enseñanza-aprendizaje, que va más allá de las fronteras de la escuela. Según Coll (2010), las TIC permiten la reformulación de la visión de la educación y la enseñanza en un sentido amplio, reconociendo la importancia creciente de otros contextos de actividad y de otros agentes educativos en las trayectorias individuales de aprendizaje. Estos escenarios dibujan la nueva ecología del aprendizaje.

Esta ecología del aprendizaje (Barron, 2006; Coll, 2013); obliga replantear algunos retos didácticos, para estar acorde a estos nuevos nichos de aprendizaje en los diferentes componentes que estudia y teoriza la didáctica, tales como: el papel del profesorado y sus decisiones didácticas frente a las TIC, las finalidades del currículo, el diseño de la clase, las estrategias didácticas usadas y el estudio sobre el pensamiento del profesorados respecto al uso de las TIC (Davidson y Goldberg, 2009, 2010; Miller et al., 2008; Williamson, 2013). A continuación, se plantean los retos que actualmente componen los escenarios de investigación del proyecto Didácticas Innovadoras mediadas por TIC: retos a partir de la ecología del aprendizaje (Jiménez-Becerra, 2018c), desde el cual se ha compilado un gran número de resultados de investigación.

\section{LOS RETOS DE LA INNOVACIÓN EN DIDÁCTICA CON TIC: COMPONENTES DE SU REDIMENSIÓN EN LA INVESTIGACIÓN}

La nueva ecología de aprendizaje (Coll, 2013), que lleva a analizar la redimensión de las didácticas, parte de repensar los retos y desafíos (Mominó \& Sigalés, 2016) que tiene la educación en el plano actual, en el marco de los nichos de aprendizaje existentes y que, hasta ahora, están siendo reconocidos por la escuela. Ahora, identifiquemos cada uno de ellos para poder reconocer los retos, en el plano de la investigación, que mueven el proyecto profesoral: Didácticas Innovadoras mediadas por TIC: cambios en la ecología del aprendizaje", aclarado que los mismos son textuales del proyecto de investigación anteriormente mencionado, por tal razón, son declarativos de su texto original.

Primer reto en el marco de los "recursos de enseñanza - aprendizaje": La constatación de que el aprendizaje se produce, y se producirá cada vez más, a lo largo y a lo ancho de la vida. A la puesta en relieve de la importancia creciente de unas necesidades de aprendizaje que se plantean a las personas mucho más allá de la educación básica y de los períodos de formación inicial, prácticamente a lo largo de toda la vida, se añade la aparición, de la mano sobre todo de las tecnologías digitales de la información y la comunicación (TIC) de nuevos e inéditos contextos de actividad que ofrecen a las personas oportunidades, recursos y herramientas para aprender. (Mominó \& Sigalés, 2016, p. 77).

Desde aquí es posible analizar cuál es el papel de las didácticas de los recursos y herramientas (Jiménez, I., y Escobar, C., 2016; Ortiz, M.; Jiménez-Becerra, I, 2018) para lograr responder a estos nichos potenciales de aprendizaje que son ajenos, al menos en principio, en las instituciones de educación formal. Un ejemplo es el papel en la enseñanza y aprendizaje de las redes sociales, los mundos o entornos virtuales, las comunidades virtuales de interés, práctica y de aprendizaje, los juegos en línea y, en general, los que permiten crear las TIC digitales; así como, su papel en el refuerzo de contextos tradicionales de actividad y de desarrollo, como la familia, la comunidad, las instituciones culturales, 
de ocio o el trabajo, como nichos potenciales de aprendizaje. Autores como Pagés (2002), plantean algunas reflexiones a tener en cuenta frente al alcance de la enseñanza en el marco de la didáctica, -en este caso es llamada las Ciencias Sociales- al sustentar que:

La finalidad última de la enseñanza de las ciencias sociales, de la geografía y de la historia ha de ser contrasocializadora, es decir ha de preparar al alumnado para que construya sus propios conocimientos, se ubique en su mundo y esté preparado para intervenir en él de manera democrática. Se reclama educar la coherencia entre el pensamiento y la acción a fin de garantizar que el protagonismo de los jóvenes ciudadanos y ciudadanas en la construcción del mundo personal y social se realice desde valores alternativos basados en el compromiso, la justicia, la tolerancia y la solidaridad. (p. 258).

El reto de quienes investigan este escenario problémico es generar resultados que apunten a detectar y plantear prácticas como oportunidades didácticas, para fortalecer los procesos pedagógicos más allá de las fronteras de la escuela; siendo los conocimientos adquiridos por los estudiantes en estos contextos, el pensamiento y conocimiento social los que permiten afrontar la sociedad y la ciber sociedad (Fresno, 2011). Esto implica, tomar postura frente a las Representaciones Sociales (Habermas, 1966, Jiménez-Becerra, 2018c), que poseen los maestros dentro del uso de las TIC, como recurso o herramienta de aprendizaje, y que puede estar ligada a su interpretación, experiencias o sistemas de creencias que poseen sobre las mismas. De ahí que, se convierten en un tema relevante para el investigador social que ahonda en la didáctica para la enseñanza usando las TIC

Segundo reto en el marco de los "ambientes de aprendizaje": Hacer converger múltiples lenguajes y formatos en un mismo espacio simbólico gracias a la digitalización de la información. Las TIC digitales se caracterizan por la utilización simultánea y convergente de diversos lenguajes y formatos, y la posibilidad de combinar lenguaje oral, lenguaje escrito, sonido, imágenes estáticas y en movimiento, lenguaje musical, lenguaje matemático, lenguaje lógico, sistemas de símbolos, sistemas de representación gráfica... Consecuentemente, las experiencias de aprendizaje y los aprendizajes relacionados con estas tecnologías están también, y lo estarán probablemente cada vez más en el futuro, modelados por la utilización de una multiplicidad de lenguajes y formatos como vehículo y soporte de la información y el conocimiento. (Mominó y Sigalés, 2016, p. 78).

Con el propósito de identificar cuáles son los nuevos escenarios de enseñanzaaprendizaje, en el contexto de las rutas didácticas que permitirán articular modelos pedagógicos, metodologías y estrategias que, acompañados con TIC, se articulan los nuevos nichos de aprendizaje. La idea es demostrar la sinergia curricular (Pérez, 2006), entre su pertinencia (inscrita a una dimensión de contexto y que responden a una misión y visión de una propuesta educativa) y su coherencia (que operacionalizan los retos formativos propuestos en los Proyectos Educativos Institucionales). Todo esto, desde un modelo que abarque la metodología, la evaluación, los objetivos declarativos de la enseñanza, las competencias y, por ende, la intencionalidad formativa que hacen parte del currículo. De ahí que, coherencia y pertinencia curricular impliquen una reflexión y una búsqueda 
constante de modelos didácticos capaces de ajustar la información, los productos y los servicios de la enseñanza a los intereses y necesidades individuales de los estudiantes. Esta es una expresión más de un sistema de valores, propia de las sociedades actuales y del pensamiento posmoderno, que sitúa al individuo en el centro de la organización social, de la actividad individual y colectiva. La evolución de las TIC digitales desde la llamada web 1.0 a la web 2.0, o web social, refleja con claridad esta tendencia. Es así como, quienes buscan investigar en este campo, asumen como reto plantear modelos y procedimientos que permitan definir las experiencias de aprendizaje y los aprendizajes relacionados con las tecnologías, al demostrar el modelo didáctico que operacionalizan la coherencia y la pertinencia curricular.

Tercer reto en el marco de los "componente cognitivo necesarios para el aprendizaje": El aprendizaje se orienta, y se orientará cada vez más, a la adquisición y dominio de habilidades y competencias genéricas y transversales que permiten seguir aprendiendo en una amplia gama de situaciones y circunstancias. En el marco de la nueva ecología del aprendizaje, lo que ya se sabe -los aprendizajes realizados- es importante, pero lo es aún más lo que se puede llegar a saber -los aprendizajes que se es capaz de realizar. (Mominó y Sigalés, 2016, p. 78).

El presente reto se ajusta a aquellos investigadores que desean identificar cómo los TIC aportaría-como andamiaje- al desarrollo de las destrezas y competencias en la aprehensión del conocimiento. Esto implica investigar los diversos enfoques del currículo, basado en los alcances de su aprendizaje, así como una mirada hacia las didácticas en Ciencias Sociales. Sin duda, en esta clasificación se pueden evidenciar elementos concretos y abstractos de las prácticas de enseñanza. Frente a los elementos concretos se reconocen las intenciones del currículum, por medio de la creación de escenarios y propuestas centradas en la enseñanza específica de las áreas de estudio, tales como las didácticas en Ciencias Naturales y Ciencias Sociales, también, la intención frente a la administración del currículum, los contenidos y la evaluación. En cuanto a los aspectos abstractos, se enumeran los discursos y, de manera específica, las concepciones o representaciones sociales que poseen los profesores y su influencia en los procesos de enseñanza y aprendizaje. A partir de esta lógica, legada de la Escuela de Frankfurt y la perspectiva filosófica de las ciencias, Pagès (1994) hace la clasificación de los tres tipos de currículo: el técnico, el práctico y el crítico, los cuales se refieren tanto a la enseñanza obligatoria, como a la formación del profesorado. Las investigaciones que ha dirigido sobre el tema se centran en los alcances de cada uno de estos, desde donde construye algunas reflexiones a tener en cuenta frente al alcance de la enseñanza de las Ciencias Sociales, pues:

La finalidad última de la enseñanza de las ciencias sociales, de la geografía y de la historia ha de ser contra socializadora, es decir ha de preparar al alumnado para que construya sus propios conocimientos, se ubique en su mundo y esté preparado para intervenir en él de manera democrática. Se reclama educar la coherencia entre el pensamiento y la acción a fin de garantizar que el protagonismo de los jóvenes ciudadanos y ciudadanas en la construcción del mundo personal y social se realice desde valores alternativos basados en el compromiso, la justicia, la tolerancia y la solidaridad. (Pagès, 2002, p. 258). 
Este planteamiento ha sido reforzado por autores como Adler (2008), Ross (2006), Singer (2008), quienes identifican el valor de la enseñanza y la importancia de la formación del profesorado, centrada en una misión y misión contundente: priorizar la representación social de los estudiantes y maestros (Jiménez-Becerra, 2018d), así como el valor de la experiencia y el contexto, como elementos centrales de la didáctica. El siguiente gráfico resume los retos de la investigación, en el marco de las Didácticas Innovadoras mediadas por TIC, a partir de la necesidad de demostrar su aporte para redimensionarla desde la ecología del aprendizaje. También, representa el alcance de estas prácticas de enseñanza desde la concepción curricular enfoque sociocrítico, el cual se resume desde el gráfico (Figura 1).

Figura 1. Retos de investigación del Proyecto Didácticas Innovadoras mediadas por TIC

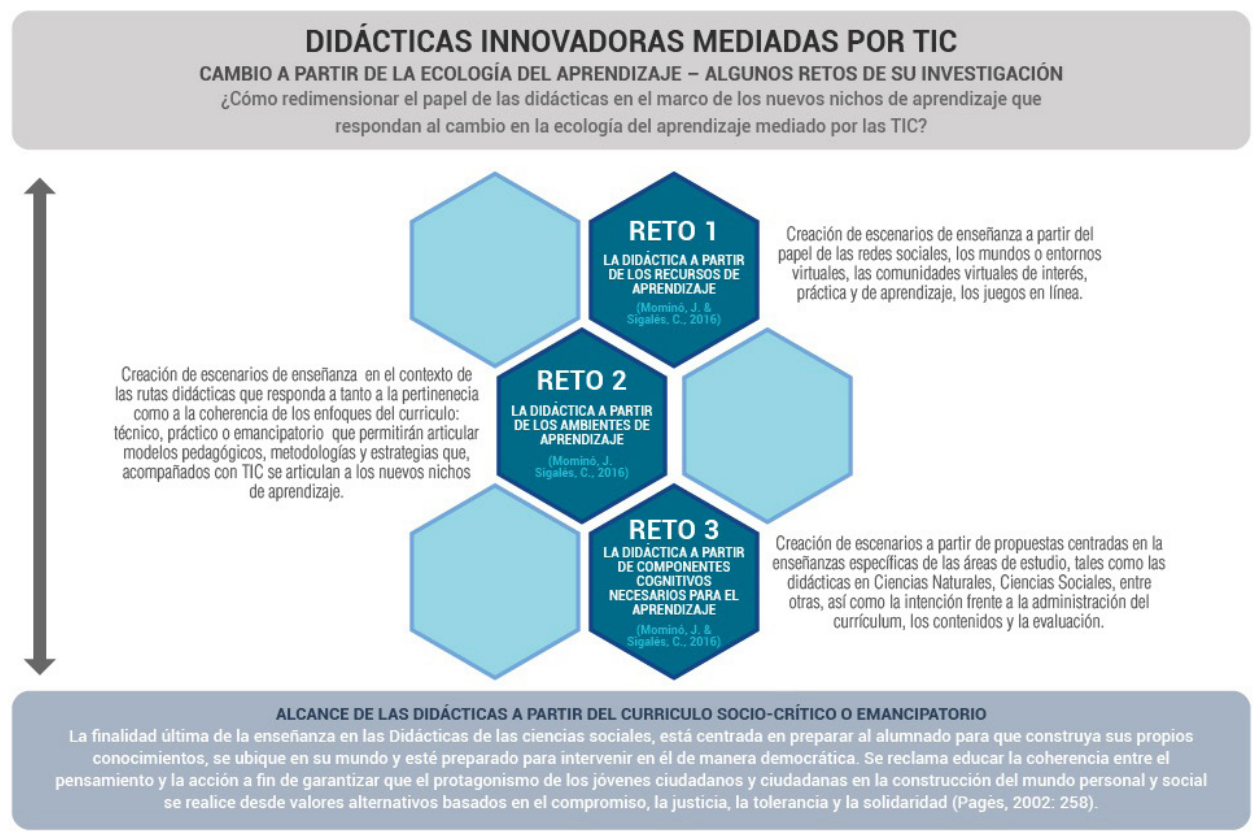

Fuente: elaboración propia

\section{DISCUSIÓN Y CONCLUSIONES: ESTUDIO DE CASO MÚLTIPLE SOBRE DIDÁCTICA, INNOVACIÓN Y TIC EN ALGUNOS CENTROS EDUCATIVOS COLOMBIANOS}

Didácticas Innovadoras mediadas por TIC es un proyecto que se originó dentro del Centro de Tecnologías -CTA- de la Universidad de La Sabana, desde el año 2015. Dichas investigaciones han impactado a cincuenta y seis instituciones educativas y algunas del sector corporativo del contexto colombiano (Jiménez, I., Fernández, O. E. y Almenárez, F. T., 2020). De igual manera, se han generado algunas publicaciones en revistas de alto 
impacto $^{3}$ y algunos capítulos de libros que han ido consolidando su teoría y práctica. La problematización del proyecto gira en tono a la pregunta de investigación: ¿Cómo redimensionar el papel de las didácticas en el marco de los nuevos nichos de aprendizaje que respondan al cambio en la ecología del aprendizaje mediado por las TIC? A partir de ahí, se ha concretado la cuestión al trasegar los siguientes objetivos específicos:

- Identificar las concepciones Didácticas usando las TIC a partir de sus representaciones sociales de las prácticas de enseñanza que poseen los maestros.

- Plantear propuestas Didácticas que apunten a uno de los retos de la redimensión de la didáctica en el marco de la ecología del aprendizaje: didácticas a partir de los recursos, de los ambientes o de los componentes cognitivos.

- Teorizar las concepciones Didácticas usando las TIC que respondan al nicho de aprendizaje investigado e implementar la propuesta didáctica que apunta a la ecología del aprendizaje, demostrando el nivel de innovación disruptiva, radical o incremental.

Didácticas Innovadoras mediadas por TIC es un proyecto adscrito al Grupo de Investigación PROVENTUS que, actualmente, posee Categoría A -una de las más altas del país- ante Departamento Administrativo de Ciencia, Tecnología e Innovación (Colciencias); entidad que regula las políticas públicas para fomentar los procesos de investigación en Colombia. Respecto a la caracterización de los participantes, se trata de maestros de regiones muy pobres de Colombia: escuelas rurales y urbanas de contextos vulnerables y con alto conflicto social. La mayoría de los casos investigados se enmarcan en tres tipos de diseño de investigación: el estudio de caso (Stake, 1975), investigación acción (Kemmis y Mc Taggart, 1988), cuando los proyectos son micro prácticas; también, en los modelos de evaluación aplicada tales como el Modelo CIPP de Sttuflebeam y Shinkfield (1987) y el Modelo Guskey (2002), cuando son de orden institucional. Todas las investigaciones coinciden en técnicas de recolección de datos como: las entrevistas, los grupos focales, el cuestionario, la observación y los análisis documentales

En el presente artículo, sintetizamos los resultados de las experiencias, basados en el estudio de caso de los tres retos propuestos, para redimensionar las prácticas de enseñanza con el uso de las TIC entre los años 2015 a 2018, en los cincuenta y seis contextos educativos ${ }^{4}$ que acopia población estudiantil de todos los niveles de educación. Además, algunas experiencias en sector corporativo (Jiménez, I., Fernández, O. E. y Almenárez, F. T., 2020) y que hicieron parte de la población seleccionada por los investigadores participantes. A continuación, se ofrece una síntesis de los resultados obtenidos, clasificados en los tres retos de innovación en Didácticas con TIC que componen el proyecto: a) en el marco de los recursos de aprendizaje: investigaciones relacionadas las estrategias de enseñanza mediado por TIC: b) en los ambientes de aprendizaje, donde se compilan investigaciones relacionadas con las concepciones y alcances del currículo mediado por TIC; y, C) propuesta sobre los componentes cognitivos necesarios para el aprendizaje, con resultados de investigaciones relacionadas con el alumno, el profesorado y el uso de las TIC.

En el ORCID de la autora del artículo se encuentra declaradas.

4 Todas las investigaciones que se articulan a estos resultados pueden ser consultadas en la Base de Datos "Intellectum" de la Universidad de La Sabana. Su espacio de acceso es: https://intellectum.unisabana.edu.co/handle/10818/9 


\subsection{INVESTIGACIONES SOBRE LOS RECURSOS DE APRENDIZAJE: RETOS RELACIONADOS CON LAS ESTRATEGIAS DE ENSEÑANZA MEDIADO POR TIC}

Los resultados de estas veinte investigaciones para redimensionar los recursos en el marco de las estrategias y metodologías se centran en identificar los fines del uso de las estrategias centradas en los estudiantes. Provocar el aprendizaje entre pares, fortalecer los procesos de comunicación e incentivar el trabajo cooperativo con el fin de mejorar el clima de convivencia en el aula, siendo insumo de trabajo las problemáticas de su contexto son parte de la intención de este reto. La estrategia didáctica implementada se ha enmarcado en enfoques de aprendizaje como la metacognición (Flavell, 1987), los estilos de aprendizaje (Kolb, 1984) y la pedagogía conceptual (De Zubiria, M. y De Zubiria, J., 1987); sumado a esto, las metodologías articuladoras como el trabajo por proyectos (Jiménez-Becerra, 2015), el desarrollo del pensamiento crítico (Dewey, 1978) y la cooperación escolar (Freinet, 1999).

Tabla 1. Investigaciones relacionadas las estrategias de enseñanza mediado por TIC

\begin{tabular}{|c|c|c|c|c|c|}
\hline $\begin{array}{l}\text { SUBCAMPOS DE } \\
\text { ANÁLISIS }\end{array}$ & \multicolumn{2}{|c|}{ AUTORES DE LOS PROYECTOS } & ALGUNOS EJEMPLOS & $\begin{array}{c}\text { ZONAS } \\
\text { COLOMBIANAS } \\
\text { DONDE SE HA }\end{array}$ & $\begin{array}{c}\text { TIPODE } \\
\text { INVESTIGACIÓN }\end{array}$ \\
\hline \multirow{10}{*}{$\begin{array}{c}\text { Referentes } \\
\text { metodológicos, } \\
\text { estrategias y/o recursos } \\
\text { de enseñanza }\end{array}$} & $\begin{array}{l}\text { Boyacá, Mejía, } \\
\text { Villalobos y } \\
\text { Valencia (2017) } \\
\text { MAPETIC }\end{array}$ & $\begin{array}{c}\text { Salamanca (2017) } \\
\text { MAPETIC }\end{array}$ & \multirow{10}{*}{$\begin{array}{l}\text { Villareal (2017) Recurso educativo digital adaptativo "el } \\
\text { rescate del reino": Una estrategia para aprender } \\
\text { permutaciones sin repetición. } \\
\text { Salamanca (2017) Implementación de entornos personales } \\
\text { de aprendizaje para fortalecer las habilidades } \\
\text { comunicativas en estudiantes de tercero de primaria del } \\
\text { colegio José Jaime Rojas. }\end{array}$} & \multirow{4}{*}{$\begin{array}{l}\text { Chía - } \\
\text { Cundinamarca } \\
\text { Bogotá - } \\
\text { Cundinamarca }\end{array}$} & \multirow{4}{*}{$\begin{array}{l}\text { Investigación } \\
\text { mixta - Aplicada } \\
\text { Modelos de } \\
\text { Evaluación } \\
\text { Guskey y CIPP } \\
\text { Investigación } \\
\text { mixta - Aplicada }\end{array}$} \\
\hline & $\begin{array}{l}\text { Campaña y Suárez } \\
\text { (2017) - MAPETIC }\end{array}$ & $\begin{array}{l}\text { Sierra (2017) } \\
\text { MIE }\end{array}$ & & & \\
\hline & $\begin{array}{c}\text { Contreras (2017) } \\
\text { MIE } \\
\end{array}$ & $\begin{array}{c}\text { Villareal (2017) } \\
\text { MAPETIC }\end{array}$ & & & \\
\hline & $\begin{array}{c}\text { Cruz (2017) } \\
\text { MIE }\end{array}$ & $\begin{array}{l}\text { Sepúlveda (2016) } \\
\text { MIE }\end{array}$ & & & \\
\hline & $\begin{array}{c}\text { Escobar (2016) } \\
\text { MIE }\end{array}$ & $\begin{array}{c}\text { Tinoco (2016) } \\
\text { MIE }\end{array}$ & & \multirow{4}{*}{$\begin{array}{l}\text { Leticia - } \\
\text { Amazonas } \\
\text { Armenia - } \\
\text { Quindío } \\
\text { Cali-Valle del } \\
\text { Cauca }\end{array}$} & \multirow{4}{*}{$\begin{array}{l}\text { Modelos de } \\
\text { Evaluación } \\
\text { Guskey y CIPP } \\
\text { Investigación } \\
\text { cualitativa - } \\
\text { Investigación } \\
\text { Básica }\end{array}$} \\
\hline & $\begin{array}{c}\text { Ibáñez (2015) } \\
\text { MIE }\end{array}$ & Tibaque (2016) & & & \\
\hline & $\begin{array}{c}\text { Moncada (2017) } \\
\text { MAPETIC }\end{array}$ & $\begin{array}{l}\text { Guzmán (2018) } \\
\text { MAPETIC }\end{array}$ & & & \\
\hline & $\begin{array}{c}\text { Pinzón } \\
\text { (2016) } \\
\text { MIE } \\
\end{array}$ & $\begin{array}{l}\text { Bruno (2018) } \\
\text { MAPETIC }\end{array}$ & & & \\
\hline & $\begin{array}{l}\text { Ortiz (2016) } \\
\text { MIE }\end{array}$ & $\begin{array}{c}\text { Domínguez, Aldana, } \\
\text { Gonzáles y Gómez } \\
\text { (2018) } \\
\text { MAPETIC }\end{array}$ & & & \multirow[t]{2}{*}{$\begin{array}{c}\text { Diseño } \\
\text { Estudio de Caso } \\
\text { Investigación } \\
\text { Acción }\end{array}$} \\
\hline & $\begin{array}{c}\text { Orozco (2016) } \\
\text { MIE }\end{array}$ & $\begin{array}{c}\text { González y Villareal } \\
\text { (2018) } \\
\text { MAPETIC }\end{array}$ & & & \\
\hline
\end{tabular}

Fuente: elaboración propia.

Los resultados encontrados demuestran que las TIC han favorecido el aprendizaje por medio de herramientas, como los metaversos de Second Life o estrategias para trabajar las problemáticas de contexto, por medio de recursos como la Realidad Aumentada (RA). Desde ahí, es posible generar experiencias de simulación centradas en problemáticas sociales, donde los estudiantes pueden experimentar situaciones reales gracias a los espacios de trabajo virtual que se asemejan a estas. Las experiencias de aprendizaje buscan que los estudiantes puedan actuar y tomar decisiones, sin las consecuencias del mundo real, pero con la ventaja de poder reflexionar dichas acciones y plantear alternativas de resolución diversas para fortalecer, de manera experiencial, su conocimiento social. 


\subsection{INVESTIGACIONES SOBRE LOS AMBIENTES DE APRENDIZAJE: RETOS RELACIONADAS CON LAS CONCEPCIONES Y ALCANCES DEL CURRÍCULO MEDIADO POR TIC}

El proyecto de investigación en Didácticas y TIC ha compilado ocho investigaciones cuya intención es generar aplicación de la didáctica sociocrítica (Pagés, 1994), tales como proceso de intersubjetividad, humanización, aproximación a la realidad y la problematización de contenidos en conexión con los conflictos sociales. Es así como el aprovechamiento del tiempo libre, el aporte a la formación en valores, la elección vocacional, la resolución de conflictos son temáticas abordadas en este tipo de investigaciones.

Tabla 2. Investigaciones relacionadas con las concepciones y alcances del currículo mediado por TIC

\begin{tabular}{|c|c|c|c|c|c|}
\hline $\begin{array}{l}\text { SUBCAMPOS DE } \\
\text { ANÁLISIS }\end{array}$ & \multicolumn{2}{|c|}{ AUTORES DE LOS PROYECTOS } & ALGUNOS EJEMPLOS & $\begin{array}{l}\text { ZONAS } \\
\text { COLOMBIANAS } \\
\text { DONDE SE HA } \\
\text { IMPLEMENTADO }\end{array}$ & $\begin{array}{l}\text { TIPO DE } \\
\text { INVESTIGACIÓN } \\
\text { Y DISEÑOS }\end{array}$ \\
\hline $\begin{array}{l}\text { Desarrollo de proyectos } \\
\text { institucionales } \\
15 \text { investigaciones }\end{array}$ & \multicolumn{2}{|c|}{ Todos los de MAPETIC del año 2018} & $\begin{array}{l}\text { Aldana (2018) Estrategias didácticas mediadas por lego } 0^{8} \\
\text { mindstorms }{ }^{8} \text { para desarrollar el pensamiento lógico para la } \\
\text { programación en los estudiantes de la facultad de } \\
\text { ingeniería de la Universidad del Quindío }\end{array}$ & \multirow{4}{*}{$\begin{array}{c}\text { Bogotá- } \\
\text { Cundinamarca } \\
\text { Leticia- } \\
\text { Amazonas } \\
\text { Armenia- } \\
\text { Quindío }\end{array}$} & \multirow{3}{*}{$\begin{array}{c}\text { Investigación } \\
\text { mixta - Aplicada } \\
\text { Modelos de } \\
\text { Evaluación } \\
\text { Guskey y CIPP } \\
\text { Investigación } \\
\text { mixta - Aplicada } \\
\text { Modelos de } \\
\text { Evaluación } \\
\text { Guskey y CIPP }\end{array}$} \\
\hline \multirow{2}{*}{$\begin{array}{l}\text { Material curricular } \\
2 \text { investigaciones }\end{array}$} & \multirow{2}{*}{\multicolumn{2}{|c|}{$\begin{array}{l}\text { Casallas (2017) } \\
\text { MIE } \\
\text { o, Torres y Moreno (2017) } \\
\text { MIE }\end{array}$}} & $\begin{array}{l}\text { Casallas (2017) Influencia de un entorno personal de } \\
\text { aprendizaje en procesos de búsqueda, selección y }\end{array}$ & & \\
\hline & & & $\begin{array}{c}\text { evaluación de información para los proyectos de } \\
\text { investigación de los estudiantes del curso } 1002 \text { del colegio } \\
\text { Marco Antonio Carreño Silva IED. }\end{array}$ & & \\
\hline $\begin{array}{c}\text { Conceptos claves de la } \\
\text { organización del } \\
\text { currículo } \\
1 \text { investigación } \\
\end{array}$ & \multicolumn{2}{|c|}{$\begin{array}{l}\text { Fonseca (2015) } \\
\text { MIE }\end{array}$} & $\begin{array}{l}\text { Fonseca (2015) El currículo procedimental: Una experiencia } \\
\text { de aula con el apoyo de un material educativo multimedia } \\
\qquad \text { TIC }\end{array}$ & & $\begin{array}{l}\text { Guskey y CIPP } \\
\text { Investigación } \\
\text { cualitativa - }\end{array}$ \\
\hline \multirow{2}{*}{$\begin{array}{l}\text { Proyectos transversales }{ }^{1} \\
\quad 4 \text { investigaciones }\end{array}$} & $\begin{array}{l}\text { Mantilla (2018) } \\
\text { MAPETIC }\end{array}$ & $\begin{array}{l}\text { Bedoya y Castro } \\
\text { (2018) MAPETIC }\end{array}$ & \multirow{2}{*}{$\begin{array}{c}\text { Chivatá y Torres (2018) Implementación de un programa de } \\
\text { aprovechamiento positivo del tiempo libre mediado por TIC } \\
\text { en los estudiantes de la básica secundaria y media de la } \\
\text { Institución Educativa Fonquetá del municipio de Chía, } \\
\text { Cundinamarca. }\end{array}$} & \multirow[t]{2}{*}{$\begin{array}{l}\text { Cali - Valle del } \\
\text { Cauca }\end{array}$} & $\begin{array}{l}\text { Investigación } \\
\text { Básica } \\
\text { Diseño }\end{array}$ \\
\hline & $\begin{array}{l}\text { Venegas y Rojas } \\
\text { (2018) MAPETIC }\end{array}$ & $\begin{array}{l}\text { Chivatá y Torres } \\
\text { (2018) MAPETIC }\end{array}$ & & & $\begin{array}{l}\text { Estudio de Caso } \\
\text { Investigación } \\
\text { Acción }\end{array}$ \\
\hline
\end{tabular}

Fuente. Elaboración propia.

Los resultados de estas investigaciones dejan entrever que las TIC han aportado a los principales alcances del currículo sociocrítico, desde la creación de contenidos en plataformas como Moodle (Modular Object-Oriented), Dynamic Learning Environment (Entorno de Aprendizaje Dinámico Orientado a Objetos y Modular) descritos por JiménezBecerra, I., Salamanca, L. \& López, L. (2018), o Modelo Canvas o Bussiness Model Canvas, acompañadas de actividades con herrameintas TIC que robustecen los ambientes de aprendizaje. Al respecto, Pagés (1994), opina que los alcances de este tipo destacan la importancia de caracterizar las habilidades o capacidades propias del pensamiento social. Esto quiere decir que fomentan la curiosidad, capacidad para considerar y buscar soluciones alternativas y originales a los problemas, que debe conocer las razones para estudiar determinadas temáticas y bajo determinada manera, dentro de situaciones concretas.

Los procesos de aprendizaje se complementaron, tanto en los ambientes como en el uso de los recursos, desde la construcción reflexiva de las unidades didácticas que evidencian, tanto la ruta como los resultados de aprendizaje esperados. Así, las áreas de estudio de mayor virtualización fueron matemáticas e inglés, pues el profesorado presentaba una gran preocupación por las dificultades en algunas habilidades de aprendizaje. Como limitaciones se reconoce la poca dotación de equipos y tecnologías, así como la ausencia 
de conectividad. Estos resultados fueron parametrizados como variables extrañas que limitaron la apropiación de conocimiento y el desarrollo de competencias en los grupos participantes.

4.3. INVESTIGACIONES SOBRE LOS COMPONENTE COGNITIVO NECESARIOS PARA EL APRENDIZAJE: INVESTIGACIONES RELACIONADAS CON EL ALUMNO, EL PROFESORADO Y EL USO DE LAS TIC

Los nueve proyectos desarrollados aquí apuntar hacia la cualificación de las habilidades de pensamiento. Al respecto, la Taxonomía de Bloom (1979), señala que son las habilidades que permiten al individuo adquirir y desarrollar pensamientos y conocimientos nuevos. Estas se clasifican en dos órdenes: las básicas que facilitan la adquisición del conocimiento; y, las superiores relacionadas con la calidad y la aplicación del aprendizaje.

Tabla 3. Investigaciones relacionadas con el alumno, el profesorado y el uso de las TIC

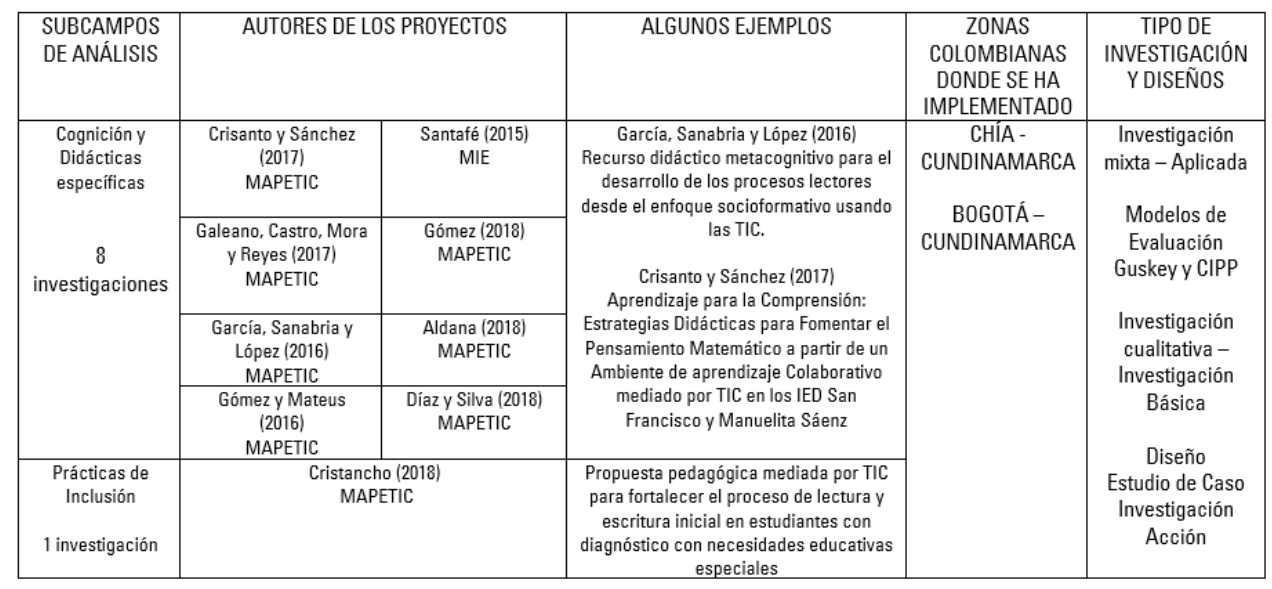

Fuente. Elaboración propia.

El reto central de estas investigaciones está focalizado en replantear la enseñanza de los contenidos programáticos más allá del aprendizaje memorístico recordando que el mismo será obsoleto en unos años; en cambio, las habilidades de pensamiento, una vez se adquieren, permanecerán con los estudiantes toda su vida. Esto también implica la preocupación por abordar prácticas de enseñanza capaces de generar procesos de inclusión, como retos particulares de la enseñanza. En este orden de ideas, las experiencias de aprendizaje con el uso de las TIC (Jiménez-Becerra, 2018b), para trabajar en contextos de problemas en escenarios virtuales, han facilitado el fortalecimiento de una variedad de categorías de aprendizaje de orden superior con los estudiantes, tales como: reflexión, razonamiento, planteamiento de problemas, solución de problemas y toma de decisiones. Como elemento emergente de estas investigaciones, en el marco del profesorado y la representación social del uso de la TIC, plantean las intranquilidades que se construyen 
en la creencia que el estudiante puede ir minimizando su motivación frente al aprendizaje, por el sobre uso o abuso de estas herramientas. Por el contrario, desde los resultados de las encuestas de percepción, se observa que un factor clave para mantener la motivación hacia el aprendizaje depende, no tanto del tiempo de uso de las TIC, sino de la calidad y naturaleza de las actividades de aprendizaje desarrolladas; lo que demuestra la riqueza de las actividades de aprendizaje, más allá del uso instrumental de las TIC.

A modo de conclusión se puede afirmar que, a partir de las investigaciones reseñadas, es posible concluir que la reflexión sobre el ejercicio de las didácticas en el contexto de la nueva ecología del aprendizaje (Jiménez-Becerra, I. 2018c) permite a los maestros investigadores debatir y cuestionar su capacidad para innovar en las prácticas de enseñanza. Esto implica responder a los interrogantes planteados por Mominó y Sigalés (2016), al afirmar que:

Hay que añadir aún todas las cuestiones relacionadas con los cambios necesarios para hacer frente a los desafíos desde las propias instituciones de educación formal y escolar, tanto en el plano de la investigación como de la innovación. Por ejemplo, ¿cómo afrontar la personalización del aprendizaje?, ¿mediante qué estrategias?, ¿cómo conocer y llevar a las instituciones de educación formal las experiencias de aprendizaje -y no solo los aprendizajes- que los alumnos y estudiantes tienen en otros contextos?, ¿cómo contribuir desde estas instituciones a conformar trayectorias individuales de aprendizaje enriquecedoras? (p. 78).

A partir de lo anterior, es posible afirmar que los desafíos del maestro, en sus prácticas de enseñanza, no sólo se sitúan en el ámbito de las políticas educativas, sino que apelan a la investigación y la innovación. En consecuencia, los investigadores, profesores y los profesionales de la educación debemos asumir retos y actuar acorde a las situaciones donde las TIC (Jiménez-Becerra, 2015) puedan a ser una estrategia significativa, tal y como lo evidencia estos resultados de investigación. En cuanto a la intención de redimensionar el papel de las didácticas, en el marco de los nuevos nichos de aprendizaje que respondan al cambio en la ecología del aprendizaje mediado por las TIC, se retoma la postura planteada por autores como Díaz, y Segovia (2015), quienes plantea que está se compone de tres cimientos, a saber: "la innovación mediada por TIC, la educación como práctica social innovadora y las TIC en las prácticas innovadoras" (p.13). Por otro lado, los resultados de investigación compilados en más de cincuenta contextos colombianos, han planteado experiencias de innovación en didáctica con mediación TIC desde tres ejes: el primero, las TIC y Educación, a partir de las tendencias con TIC y las tendencias pedagógicas/educativas, centradas en metodologías, ambientes y materiales que componen los fines de la educación, los alcances de los aprendizajes y el aporte al fortalecimiento de procesos cognitivos, tales como el pensamiento crítico (Dewey, 1978) o el pensamiento social (Santisteban y Pagés 2006, Jiménez-Becerra, I. 2020). En un segundo lugar, el eje de innovación referido a la naturaleza o al impacto de esta innovación que, tal como lo plantea Bocconi, Kampylis \& Punie, (2013), puede ser de corte disruptiva, radical e incremental. Por lo anterior, las propuestas de Innovación Educativa mediada por TIC están directamente relacionadas con productos, servicios, modelos y procesos que se han ido consolidando y que aportan a los procesos de transformación y mejoramiento de una problemática educativa particular, en consonancia con lo propuesto por los tres retos de investigación del proyecto presentado. 
Otro aspecto importante para tener en cuenta es el paradigma que busca alcanzar las diferentes investigaciones cuyo objeto de estudio es la "Educación y TIC" (JiménezBecerra, I. 2018a), aspecto que se refleja en los diseños de investigación propuesto. Como fue presentado en el mapeo de resultados por restos de la investigación de las Didácticas con TIC, las investigaciones que giraron en torno a la Investigación-Acción, buscaron generar innovación a partir de diversas micro prácticas centrando el interés en el papel del profesorado y la reflexión de las prácticas de enseñanza con TIC. Respecto al estudio de caso, estos investigadores centraron su interés en comprender los fenómenos de estudio en el marco de los problemas educativos y de la enseñanza, donde las TIC podrían ser un andamiaje posible que aporta a la solución, comprendiendo su papel en el ejercicio de la enseñanza-aprendizaje. Finalmente, las investigaciones que giraron en los modelos de evaluación aplicada giraron en investigaciones de alcance institucional, evaluando programas o prácticas transversales para plantear estrategias innovadoras que aportaran a la cualificación de los proyectos institucionales. Los resultados finales han respondido a los objetivos específicos de la investigación centrados identificar, plantear y teorizar las concepciones Didácticas usando las TIC que respondan al nicho de aprendizaje investigado e implementar la propuesta didáctica que apunta a la ecología del aprendizaje, demostrando el nivel de innovación disruptiva, radical o incremental, lo cual ha consolidado su objetivo general que es la redimensión el papel de las didácticas en el marco de los nuevos nichos de aprendizaje que respondan al cambio en la ecología del aprendizaje mediado por las TIC.

\section{REFERENCIAS BIBLIOGRÁFICAS}

Adler, S. (2008). The education of social studies teachers. Handbook of research in social studies education. New York: Routledge, pp. 329 - 350.

Barron, B. (2006). Interest and self-sustained learning as catalysts of development: A learning ecologies perspective. Human Development, 49, pp. 193-224.

Belloch, C. (2012). Las Tecnologías de la Información y Comunicación en el aprendizaje. Recuperado el18/11/2017

Bocconi, S., Kampylis, P., \& Punie, Y. (2013). Innovating teaching and learning practices: Key elements for developing Creative Classrooms in Europe. eLearning Papers, Special edition 2013, 8-20.

Coll, C. (2013). El currículo escolar en el marco de la nueva ecología del aprendizaje. Aula de innovación educativa, pp. 31-36.

Coll, C. (2010). Enseñar y aprender, construir y compartir: procesos de aprendizaje y ayuda educativa. En C. Coll (Coord.), Desarrollo, aprendizaje y enseñanza en la educación secundaria. Barcelona: Graó, pp. 31-61.

Churches, A. (2009). Taxonomía de Bloom para la Era Digital. Recuperado de: HYPERLINK "http://www.eduteka.org/TaxonomiaBloomDigital.php"

Davidson, C., y Goldberg, D. (2009). The future of learning. Institutions in a digital age. Cambridge, Massachusetts: The MIT Press. [Archivo en línea]. Recuperado de: https://mitpress.mit.edu/ books/future-learning-institutionsdigital-age.

De Zubiria, M. y De Zubiria, J. (1987). Fundamentos de pedagogía conceptual. Una propuesta curricular para la enseñanza de las Ciencias Sociales para pensar. Bogotá: Plaza\&Janes.

Del Fresno, M. (2011). Netnografía. Barcelona: Editorial UOC.

Dewey, J. (1978). Democracia y educación: una introducción a la filosofía de la educación (8a ed.). Buenos Aires: Losada. 
Díaz, D., Segovia, Y. (2015). Documento Maestro Maestría en Innovación Educativa mediada por TIC. Centro de Tecnologías para la Academia. Universidad de La Sabana. Chía, Colombia.

Flavell, J. H. (1987). Speculations about the nature and development of metacognition. En F. E. Weinert y R. H. Kluwe (compiladores), Metacognition, Motivation and Understanding. Hillsdale: NJ: Erlbaum

Freinet, C. (1999). La Escuela Moderna francesa. Una pedagogía moderna de sentido común. Las invariantes pedagógicas. Madrid: Ediciones Morata.

Guerrero, M. (2014). Metodologías activas y aprendizaje por descubrimiento. Las TIC y la educación. Editorial: Campuseducación.com.

Guskey, T. (2002). Does it make a difference?: Evaluating professional development. Educational Leadership, 59(6), pp. 45-51

Habermas, J. (1966). Teoría y praxis: ensayos de filosofía social. Buenos Aires: Sur.

JISC. (2012). Learning in a digital age. [Archivo en línea]. (Consultado el 30/09/2013). Recuperado de: http://www.jisc.ac.uk/whatwedo/programmes/elearning/digilifelong.aspx>.

Jiménez-Becerra, I. (2015). Pedagogía de la creatividad viable: un camino para potencializar el pensamiento crítico. Opción, 31(2), 632-653. Recuperado de https://www.redalyc.org/ pdf/310/31045568035.pdf

Jiménez-Becerra, I., y Escobar, C. (2016) Uso didáctico del videojuego educativo para la enseñanza de las ciencias sociales: un estado del arte. Paideia, 11(58), 53-70.

Jiménez-Becerra, I., Salamanca, L., \& López, L. (2018). Implementación de Entornos Personales de Aprendizaje para fortalecer las habilidades comunicativas. Revista de La Universidad Pedagógica Nacional. Facultad de Humanidades, 47(11), 119-132

Jiménez-Becerra, I.; Ortiz, V. (2018). Efecto de un recurso educativo digital adaptativa en las habilidades espaciales de estudiantes de secundaria Espacios ISSN: 0798-1015 Editorial Asociación de Profesionales y Técnicos del CONICIT. v.39 fasc./a p.1 - 7 ,2018, DOI: 00000003-4263-1970

Jiménez- Becerra, I. (2018a). Didáctica y TIC: un escenario de la investigación educativa. En Jiménez, I. (2018) Informática educativa (pág. 16). Chía: Universidad de la Sabana.

Jiménez-Becerra, I. (2018b). Informática Educativa: origen, naturaleza y perspectiva de su investigación (ECO Ediciones y Universidad de La Sabana, Ed.). Bogotá.

Jiménez-Becerra, I. (2018c). Didácticas Innovadoras mediadas por TIC: retos a partir de la ecología del aprendizaje. Chía, Cundinamarca

Jiménez-Becerra, I. (2018d). Representación Social del Conflicto Colombiano en los maestros de Básica Primaria. Universidad Autónoma de Barcelona.

Jiménez-Becerra, I., Fernández, O. E. y Almenárez, F. T. (2020). Diseño pedagógico adaptativo para el desarrollo de MOOC: una estrategia para el desarrollo de competencias en contextos corporativos. Revista Electrónica de Investigación Educativa, 22, e16, 1-17. https://doi. org/10.24320/redie.2020.22.e16.2192

Jiménez-Becerra, I. (2020). Modelo didáctico tecnosocial: una experiencia de educación para la ciudadanía con jóvenes universitarios desde el estudio de los conflictos sociales. El Futuro del Pasado, 11, pp. 637-658. https://doi.org/10.14516/fdp.2020.011.021.

Kemmis, S. y McTaggart, R. (1988). Cómo planificar la investigación-acción. Barcelon: Editorial Laertes.

Kennedy, G., Judd, T. S., Churchward, A., Gray, K., y Krause, K.-L. (2008). First year students' experiences with technology: Are they really digital natives? Australasian Journal of Educational Technology, 24(1), 108-122

Kolb, D. (1984). Experiential Learning: Experience as the source of learning and development. New Jersey: Prentice Hall.

Miller, R. et al. (2008). School's Over: Learning Spaces in Europe in 2020: An Imagining Exercise on the Future of Learning. Joint Research Center. European Commission. [Archivo en línea]. 
(Consultado el 23/08/2013). Recuperado de: ftp://ftp.jrc.es/pub/EURdoc/ JRC47412.pdf.

Moscovici, S. (2000). Social representations. Explorations in social psychology. Cambridge: Polity Press

Mominó, J., y Sigalés, C. (2016) El impacto de las TIC en la Educación: Más allá de las promesas. Cataluña. Editorial UOC Ediciones.

Miller, R. et al. (2008). School's Over: Learning Spaces in Europe in 2020: An Imagining Exercise on the Future of Learning. Joint Research Center. European Commission. [Archivo en línea]. (Consultado el 23/08/2013). Recuperado de: ftp://ftp.jrc.es/pub/EURdoc/ JRC47412.pdf.

Moscovici, S. (2000). Social representations. Explorations in social psychology. Cambridge: Polity Press

Mominó, J. \& Sigalés, C. (2016) El impacto de las TIC en la Educación: Más allá de las promesas. Cataluña. Editorial UOC Ediciones.

Pagés, J., \& Satisteban, A. (2006). ¿Qué saben y qué debería saber de política los alumnos al finalizar la enseñanza obligatoria? Una investigación sobre Educación Cívica y formación democrática de la ciudadanía. La Educación para la Ciudadanía. Universidad Atónoma de Barcelona.

Pagès, J. (2002). Enseñar y aprender Ciencias Sociales, geografía e historia de la educación secundaria. Líneas de Investigación en didácticas de las Ciencias Sociales. En: Benejam, P. \& Pagès, J. (coords) , pp. 209-226.

Pagès, J. (2000). La formación inicial del profesorado para la enseñanza del patrimonio histórico y de la historia. Treballs d'Arqueologia, 6, pp. 207 - 217

Pagès, J. (1994). La didáctica de las Ciencias Sociales, el currículum y la formación del profesorado. Signos Teoría y Práctica de la Educación, 5(13), pp. 38 - 51.

Pérez J. (2000). La evaluación de programas educativos: conceptos básicos, planteamientos generales y problemática. Revista de Investigación Educativa, 18(2), pp. 261-287. Recuperado de: http:// www.doredin.mec.es/documentos/007200230097.pdf.

Ruíz, F. (2016) Escenarios de futuro y transformación del sistema educativo. En: Mominó, J; Sigalés, C. (2016) El impacto de las TIC en la Educación: Más allá de promesas. Editorial UOC Ediciones. Barcelona, España.

Santisteban, A. y Pagés, J. (2006). La enseñanza de la historia en la educación primaria. In: Casas, M. y Tomàs, C. (Coord.). Educación primaria. Orientaciones y recursos. Barcelona: Wolters Kluwer Educación, pp. 129-160.

Stake, R. (1974). Nine approaches to educational evaluation. Urbana, il, University of Illinois.

Stufflebeam, D., y Shinkfield, A. (1987). Evaluación Sistemática. Guía teórica y práctica. Madrid: MEC - Paidós.

San Martín, A. (1991). Materiales curriculares: cómo elaborarlos, seleccionarlos y usarlos (4a Ed.). Barcelona: Grao de Servies Pedagogics

Redecker, Ch., Leis, M., Leenderse, M., Punie, Y., Gijsbers, G., Kirschner, P., Stoyanov, S., y Hoogveld, B. (2011). The future of Learning: Preparing for Change. JRC-Joint Research Centre. Institute for Prospective Technological Studies. European

Ross, E. W. (2006). The social studies curriculum. New York: SUNY Press.

Singer, A. (2008). Social studies for secondary schools: Teaching to Learn, Learning to Teach (3rd ed.). New York: Taylor y Francis.

Williamson, B. (2013). The future of the Curriculum. School knowledge in the digital age. Cambridge, Massachusetts: The MIT Press. Disponible en línea (consultado el 23/08/2013): <http://mitpress. mit.edu/books/future-curriculum>. 
